\title{
Evolución de la consejería en rehabilitación en Puerto Rico: Esfuerzos organizacionales y proyectos legislativos
}

\section{Evolution of Rehabilitation Counseling in Puerto Rico: Organizational and Legislative Efforts}

\author{
Ángel A. Villafañe Santiago ${ }^{7}$ \\ Universidad de Puerto Rico, Recinto de Río Piedras \\ Puerto Rico \\ angelpr39@gmail.com \\ José Lionel Velázquez Altagraciaª \\ Universidad de Puerto Rico, Recinto de Río Piedras \\ Pueto Rico \\ velazquezjoselionel@ymail.com \\ Maribel Báez Lebrón ${ }^{3}$ \\ Universidad de Puerto Rico, Recinto de Río Piedras \\ Puerto Rico \\ mbl1385@yahoo.com
}

\footnotetext{
Doctorado en Educación con concentración en Consejería de la Universidad Interamericana de Puerto Rico y maestría en Consejería en Rehabilitación de la Universidad de Puerto Rico. Bachillerato en Artes con concentración en Trabajo Social de la Universidad Central de Bayamón, Puerto Rico; Certificación en Evaluación Vocacional de la Universidad Interamericana de Puerto Rico y Certificación en Asistencia Tecnológica del Instituto Fillius de la Universidad de Puerto Rico. Catedrático Asociado del Departamento de Consejería para el Desarrollo Estudiantil (DCODE) de la Universidad de Puerto Rico. Investigador en el área de la violencia de género y otros temas. Publicaciones sobre violencia intrafamiliar, VIH/SIDA, hijos adultos de padres/madres alcohólicos (HADA), estudiantes internacionales y asistencia tecnológica, entre otros. Experiencia dictando cursos graduados y subgraduados relacionados a la conducta humana. Ex miembro de la Junta Examinadora de Consejeros en Rehabilitación de Puerto Rico del Departamento de Salud (JECR), del Comité de Investigación para la Seguridad del Sujeto Humano en la Investigación y del Colegio de los Profesionales de la consejería en rehabilitación. Premio ACER a la Excelencia Profesional (1994) y Mary E. Switzer (2007) otorgado por la Asociación Puertorriqueña de Rehabilitación, Puerto Rico.
}

2 Maestría en Consejería en Rehabilitación, Certificación en Evaluación Vocacional y Bachillerato en Artes, concentración en Ciencias Políticas de la Universidad de Puerto Rico. Experiencia profesional: 1971-2006 en la Administración de Rehabilitación Vocacional en puestos de consejería en rehabilitación, administración y supervisión. Ex miembro de la Junta Examinadora de Consejeros en Rehabilitación del Departamento de Salud. Miembro del Comité Asesor para la Maestría en Consejería en Rehabilitación de la Universidad Central de Bayamón; Consejo Estatal de Planificación de Salud Mental y primer presidente del Colegio de los Profesionales de la Consejería en Rehabilitación de Puerto Rico Inc. Exmiembro de la Asociación Puertorriqueña de Rehabilitación; de la Asociación Puertorriqueña de Evaluadores Vocacionales, de la National Rehabilitation Association, y de la Vocational Evaluation and Work Adjustment Association, Virginia, USA. Premio Mary E. Switzer (1995), otorgado por la Asociación Puertorriqueña de Rehabilitación y premio Consejero en Rehabilitación del Año (2004), por la Asociación de Consejeros en Rehabilitación. Premio Trayectoria (2009) por la Asociación de Estudiantes de Consejería en Rehabilitación de la Universidad de Puerto Rico. Publicaciones: Salud mental laboral del profesional de la consejería en rehabilitación en Puerto Rico.

3 Doctorado en Psicología con especialidad en Psicología Industrial Organizacional y Maestría en Consejería en Rehabilitación de la Universidad de Puerto Rico. Especialidad en Rehabilitación Psiquiátrica de la Universidad de Pittsburgh. Certificación en Métodos Alternos de Negociación (Mediación) de la Facultad de Derecho de la Universidad Interamericana de Puerto Rico y Bachiller en Psicología de la Universidad de Puerto Rico, Recinto de Río Piedras. Catedrática Auxiliar de la Escuela Graduada de Consejería en Rehabilitación (CORE) de la Universidad de Puerto Rico donde su función principal es formar a los futuros profesionales de la Consejería en Rehabilitación y coordinar prácticas e internados. Forma parte del Grupo de Estudios del Trabajo, adscrito al Centro de Investigaciones Sociales (GET) y Grupo de Psicología Crítica del Trabajo y las Organizaciones (GPCT), de la Universidad de Puerto Rico, Recinto de Río Piedras. Miembro de la Junta Examinadora de Consejeros en Rehabilitación de Puerto Rico del Departamento de Salud de Puerto Rico (JECR). Experiencia como investigadora en el área de la adherencia al tratamiento, ideologías del trabajo sobre la discapacidad, transformaciones del trabajo y precariedad, entre otros. Experiencias dictando cursos graduados relacionados a la consejería en rehabilitación. 
URL: http://www.una.ac.cr/educare

CORREO: educare@una.cr

Recibido 4 de julio de 2013 • Corregido 8 de agosto de 2013 • Aceptado 15 de agosto de 2013

Resumen. El propósito de este trabajo es presentar: a) los esfuerzos históricos, organizacionales y legislativos que han contribuido al desarrollo de la profesión de consejería en rehabilitación, b) cómo estos esfuerzos han aportado al desarrollo de la identidad profesional de los consejeros y consejeras en rehabilitación y c) cómo estos acontecimientos han contribuido a expandir el desarrollo de programas y la prestación de servicios de calidad para las personas con necesidades especiales en Puerto Rico. A través de la historia oral y la revisión de bibliografía se reconoce la importancia de la creación de la legislación federal que promovió el primer programa de servicios para personas con necesidades especiales en los Estados Unidos y Puerto Rico. Estos mandatos legislativos también han promovido el desarrollo de la profesión de consejería en rehabilitación en el siglo XX y el desarrollo de las organizaciones profesionales determinantes en el establecimiento de la profesión. Se ilustra cómo, a finales de 1960 en Puerto Rico, la creación de la Asociación de Consejeros en Rehabilitación fue fundamental para la transformación de la profesión y la creación del Colegio de los Profesionales de la Consejería en Rehabilitación de Puerto Rico, a principios del siglo XXI, así como la contribución de un grupo de personas relacionadas con esta. Se presentan, además, los esfuerzos legislativos posteriores y la aprobación de nuevos mandatos, incluyendo la modificación de la Ley de Salud Mental de Puerto Rico para incorporar a este profesional entre los profesionales que prestan servicios a las personas con problemas de salud mental en el país. Después de esta revisión teórica e histórica se concluye que, si bien la profesión aún necesita más mercadeo, es una profesión de vanguardia que se ha ido actualizando con el tiempo, lo que garantiza el compromiso profesional de seguir ofreciendo servicios de calidad a las personas con necesidades especiales.

Palabras claves. Consejería en rehabilitación, legislación, historia, profesionalización.

Abstract. The purpose of this paper is to explain a) the historical, organizational, and legislative efforts that have contributed to the development of the Rehabilitation Counseling profession, b) how these efforts have contributed to the development of the professional identity of the rehabilitation counselors and c) how these events have contributed to the development of programs and the provision of quality services to people with special needs in Puerto Rico. Through oral history and literature review, it is possible to recognize the importance of the establishment of the federal legislation that promoted the first program of services to people with special needs in the United States and Puerto Rico. Those legislative mandates have also promoted the development of the Rehabilitation Counseling profession in the 20th Century and the development of professional organizations that have been determinant for the professionalization. This paper illustrates how at the end of 1960, in Puerto Rico, the creation of the Rehabilitation Counselors Association (Asociación de Consejeros en Rehabilitación, ACER by its Spanish acronym) was critical for the transformation of the profession and for the creation of the Puerto Rico's Professional Society of Rehabilitation Counselors (Colegio Profesional de los Consejeros en Rehabilitación de Puerto Rico) at the beginning of the 21st Century. The study refers to the subsequent legislative efforts and the approval of new mandates as the amendment to the Puerto Rican Law of Mental Health made to include the Rehabilitation Counseling professionals among the professionals authorized to provide such services to people with mental health conditions in the country. After this theoretical historical review, it is concluded that although more marketing is required in terms of promoting the profession, this innovative profession has kept up-to-date over time, and that is a guarantee of the professional commitment to continue providing quality services to the persons with special needs.

Keywords. Rehabilitation counseling, legislation, history, professionalism. 


\section{Introducción}

La consejería en rehabilitación es una especialización dentro de la consejería que comenzó hace unos 93 años en los Estados Unidos de América (EUA). Riggar y Maki (2004) la definen como un proceso sistemático que asiste a las personas con necesidades especiales (referidas a personas con impedimentos, diversidad funcional o discapacidad) para lograr sus metas personales, desarrollo de la carrera, ocupación y vida independiente a través del proceso de consejería. La función primaria de los profesionales de la consejería en rehabilitación es asistir a las personas con necesidades especiales a lidiar con los aspectos psicosociales, vocacionales, empleo y de vida independiente. No obstante, como profesional de la consejería también puede ofrecer servicios a personas sin necesidades especiales (Ley 198, 2006).

Esta profesión se distingue de otras profesiones dentro del campo de la salud y la conducta humana -como trabajo social, psicología, orientación y consejería- porque surge por mandato de ley en EUA; y luego, en Puerto Rico, a principios de Siglo XX, para cumplir un encargo político, social y humanista durante el periodo de la Primera Guerra Mundial, donde su énfasis primordial son las personas con necesidades especiales. Tomando esto en consideración, se presenta un breve relato histórico del desarrollo de la consejería en rehabilitación dentro del contexto legislativo de los EUA y Puerto Rico; haciendo un énfasis particular en Puerto Rico, resaltando el rol protagónico de las organizaciones profesionales, los precursores y los retos del nuevo milenio.

\section{Inicio de la profesión}

La primera pieza legislativa identificada en la bibliografía es la Ley Smith-Hughes del 1917 (Ley Pública 64-347), la cual estableció la Junta Federal de Educación Vocacional para administrar los programas de rehabilitación vocacional para veteranos (Parker y Szymanski, 1998; Parker, Szymanski y Patterson, 2005; Riggar y Maki, 2004; Rubin y Roessler, 2008). La legislación promueve que la consejería en rehabilitación eventualmente esté asociada con los colegios de educación en los EUA. En el año 1918 se aprueba la Ley para la Rehabilitación del Soldado (SmithSears Act o Soldier's Rehabilitation Act), la cual crea el Programa de Rehabilitación Vocacional para Veteranos lesionados durante la guerra (Rubin y Roessler, 2008). Este programa también era administrado por la Junta Federal de Educación Vocacional (Parker, Szymanski y Patterson, 2005; Riggar y Maki, 2004; Rubin y Roessler, 2008). Posterior a estas dos leyes, en 1920 entra en vigor la Ley Smith-Fess (Ley Pública 66-236), que marca el inicio del Programa de Rehabilitación Vocacional para civiles con necesidades especiales físicas, administrado por la Junta Federal de Educación Vocacional (Parker, Szymanski y Patterson, 2005; Riggar y Maki, 2004; Rubin y Roessler, 2008). Los fondos destinados a través de esta ley podían ser utilizados para servicios de orientación vocacional, pago de educación universitaria y no universitaria, servicios de ajuste ocupacional, prótesis y colocación para empleo.

Finalmente, el Congreso de los Estados Unidos hace extensivo los beneficios de la Ley Smith-Hughes a Puerto Rico; facilitando la creación de la Junta Estatal de Instrucción Vocacional, 
URL: http://www.una.ac.cr/educare

CORREO: educare@una.cr

mediante la aprobación de la Ley 28 de Puerto Rico en el mismo año (Ley 28 de 1931). Esta extensión es posible por la relación socio política de Puerto Rico con los EUA. La pieza proveía para la creación de la Junta y disponía que a la Junta Estatal también estuviera adscrita la División de Rehabilitación Vocacional. Esta administraría el Programa de Rehabilitación Vocacional, hoy anexado a la Administración de Rehabilitación Vocacional del Departamento del Trabajo y Recursos Humanos de Puerto Rico; pero, por falta de fondos, la prestación de servicios se aplazó por cinco (5) años. La Administración de Rehabilitación Vocacional adscribe varios títulos de la Ley de Rehabilitación, según enmendada y uno de ellos es el título I conocido como el Programa de Rehabilitación Vocacional y esta es la unidad estatal designada (Ley 93-112 de 1973).

En 1936 se establece la División de Rehabilitación Vocacional al amparo del Departamento de Instrucción Pública, hoy Departamento de Educación, adscrita a la Junta Estatal de Instrucción Vocacional. Con el establecimiento del Programa de Rehabilitación Vocacional se inicia la prestación de servicios de consejería en rehabilitación en Puerto Rico a una población creciente con necesidades especiales físicas congénitas y adquiridas. Los profesionales que brindaban servicios de rehabilitación, hoy personas consejeras en rehabilitación vocacional, se les llamaba agentes de rehabilitación (E. Albizu y J. Velázquez, comunicación personal, febrero, 18, 2006). De acuerdo con estos autores, ascendían a 10 agentes de rehabilitación. Se ofrecían servicios a personas con condiciones físicas y respiratorias. Entre las condiciones físicas atendidas se encontraban personas con amputaciones, labio leporino, paladar fisurado y problemas nutricionales. Los servicios de restauración incluían cirugía, prótesis dental y terapia del habla para trabajar, después del proceso quirúrgico, con el lenguaje expresivo; particularmente en aquellas personas con labio leporino.

El desarrollo legislativo, a medida que iba pasando el tiempo, también continuó su evolución. En los EUA se aprueba la Ley Wagner-Payser (Ley Pública 73-30 del 6 de junio de 1933). Esta pieza, a diferencia de sus predecesoras, desarrolla nuevos servicios para todos los estados, incluyendo a Puerto Rico (Ley 73-30, 1933; Riggar y Maki, 2004; Rubin y Roessler, 2008). Entre los servicios que proveyó se encuentra la creación de la Oficina de Servicios de Empleo (Ley Pública 73-30, 1933). Dicha ley tiene un lenguaje y criterios específicos para la prestación de servicios de consejería y colocación a personas con necesidades especiales en las oficinas de empleo de los Estados, incluyendo los territorios como Puerto Rico.

Por disposición de la Ley Barden Lafayette de 1943, se amplía la prestación de servicios a la población de personas con trastornos mentales y retardación mental y se expanden los servicios de reconstrucción física (cirugías de remplazo de cadera, prótesis en dedos de pies y mano, facial, entre otros) y para las personas con necesidades especiales visuales (Riggar y Maki, 2004). Durante ese periodo en Puerto Rico se incluyen, en la prestación de servicios del Programa de Rehabilitación Vocacional, los servicios de psicólogos y prostetistas (E. Albizu y J. Velázquez, comunicación personal, febrero, 18, 2006). Estos autores añaden que con esta ley el componente de salud mental se fortalece y permite la inclusión del diagnóstico de epilepsia y otros servicios de restauración, además del componente de los inicios de los procesos de evaluación vocacional y adiestramiento vocacional. 
Continúan señalando que, cinco años más tarde, un evento importante se manifiesta en el desarrollo legislativo puertorriqueño con la aprobación de la Ley de Rehabilitación Vocacional de Puerto Rico, conocida como la Ley 414 de 13 de mayo de 1947. Con esta, se proveyeron las bases para la operación del Programa de Rehabilitación Vocacional Estatal y se ampliaron los servicios a personas con otras necesidades especiales que no fueran solamente físicas (Ley 414 de 1947). También se logró un pareo de fondos permanente de 80 y 20 por ciento entre los EUA y Puerto Rico, respectivamente, lo que garantizaba la prestación de servicios a largo plazo siempre y cuando el programa cumpliera con el logro de las metas programáticas. De acuerdo con la historia oral recopilada en entrevista personal realizada a Albizu y Velázquez (2006), el primer presupuesto asignado a Puerto Rico fue de $\$ 113,000$ por año fiscal.

El 3 de agosto 1954, se enmienda la Ley de Rehabilitación Vocacional de los EUA, conocida como la Ley pública 565 o Hill Burton Act, la cual constituye la primera revisión de mayor amplitud del Programa de Rehabilitación Vocacional desde 1943 (Rubin y Roessler, 2008). Esta enmienda autoriza servicios para las personas con necesidades especiales más severos y para hacer mejoras a las facilidades de los talleres vocacionales y escenarios de rehabilitación (Parker, Szymanski y Patterson, 2005). Esta ley también obliga al reclutamiento del personal más cualificado para la prestación de servicios de las personas con necesidades especiales y el desarrollo de los programas de rehabilitación (Rubin y Roessler, 2008). Con ella, se establece el modelo de prestación de servicios de rehabilitación vocacional que prevalece hasta hoy y se establece, de manera clara y precisa, que el consejero en rehabilitación es el coordinador primario de los servicios de rehabilitación vocacional dentro de esa Agencia.

Considerando la relevancia del consejero en rehabilitación en este proceso, la ley también permitió la aportación de fondos federales para adiestramiento a nivel graduado e investigación, el pago de adiestramiento para personas interesadas en formarse dentro de la disciplina y la expansión de los currículos de programas graduados de consejería en rehabilitación en las universidades y centros de adiestramientos (Riggar y Maki, 2004). Previo a esta ley, desde los años 1940, había instituciones educativas que preparaban consejeros en rehabilitación; pero después de 1954 la consejería en rehabilitación comienza a crecer, lo que promueve la profesionalización y el desarrollo de programas académicos para preparar a este profesional y le permite establecer su propia identidad profesional (American Medical Association, 2008).

En Puerto Rico los primeros cursos de consejería en rehabilitación se inician entre los años 1954 y 1955, a petición de la División de Rehabilitación Vocacional del Departamento de Instrucción Pública, a través de la Universidad de Puerto Rico. Para el 1959 se establece el diploma profesional en consejería en rehabilitación y en el 1963 se gradúa el primer grupo ( $E$. Albizu y J. Velázquez, comunicación personal, febrero, 18, 2006).

Estos autores, conjuntamente con (L. Bonilla, comunicación personal, noviembre, 112003 ) precursores de la profesión en diferentes momentos históricos de Puerto Rico, identificaron que luego de la creación del diploma profesional en Puerto Rico, el Programa de Rehabilitación Vocacional Estatal coordinó con la Universidad de Nueva York para enviar, durante los veranos, a su personal 
URL: http://www.una.ac.cr/educare

CORREO: educare@una.cr

de consejería para concluir los estudios iniciados en Puerto Rico y hacer la maestría en el área. A partir del año 1955 se inició el envío de acuerdo con el "señorío" o años de experiencia; se comenzó con los supervisores ubicados en la Oficina Central del Programa de Rehabilitación Vocacional y, posteriormente, con el personal ubicado en las oficinas regionales donde se ofrecían servicios.

El primero en acudir y terminar los estudios fue el Sr. José Martínez Pacheco, quien era sub-director del Programa de Rehabilitación Vocacional que dirigía Domingo Collazo Collazo. A este se unieron Miguel Pérez, Antonio Carrero, Luis A. Bonilla, Antonio Padilla y Armando Ramos, entre otros. Entre las féminas del grupo se destacaron Blanca Irizarry, quien estudió en New York University, entre el 1954 y 1956. Para la misma época, la señora María Cristina Rivera, estudió en la Universidad de Wisconsin, donde obtuvo una Maestría en Artes en Rehabilitación Vocacional, posteriormente tomó otros cursos para completar los requisitos de la Maestría de Consejería en Rehabilitación. La señora Elena Arroyo, por su parte, estudió por su cuenta a través de una beca en la Universidad de Virginia. En total se estima que 18 personas se beneficiaron de estas propuestas de adiestramiento (J. Velázquez, comunicación personal, julio, 8, 2006). El énfasis de los estudios se daba según el área de trabajo. El señor Luis A. Bonilla, que supervisaba una facilidad de rehabilitación, se enfocó en Investigación, Administración y Supervisión de Facilidades. Otros cursos tenían énfasis en el área de aspectos médicos y psicosociales, lo que promovía las especialidades y facilitaba la integración del consejero en rehabilitación con otros profesionales de la salud.

Finalmente, para el 1972 en Puerto Rico, queda instituido el Grado de Maestría en Consejería en Rehabilitación de la Universidad de Puerto Rico, con 55 créditos y la acreditación del Council on Rehabilitation Education (CORE) que ha mantenido de manera ininterrumpida hasta hoy. En los últimos años, otras dos escuelas graduadas se han establecido en el país con los niveles de excelencia establecidos por CORE. Estas son la Pontificia Universidad Católica de Puerto Rico, Recinto de Ponce, y la Universidad Central de Bayamón, que cuenta con un programa especializado en Evaluación Vocacional.

Es claro que estos esfuerzos académicos y legislativos de mediados del siglo XX ayudaron a que se continuara desarrollando la profesión de consejería en rehabilitación, lo que facilitó que, el 27 de mayo de 1976, se firmara la Ley 58. Esta regula la práctica de la consejería en rehabilitación en Puerto Rico y crea la Junta Examinadora de Consejeros en Rehabilitación, adscrita al Departamento de Estado. Esta ley establece que todo aspirante a ejercer la consejería en rehabilitación debe tener un grado de maestría o doctorado en consejería en rehabilitación, haber aprobado un examen de reválida y estar certificado por la Junta Examinadora de Consejeros en Rehabilitación (Ley 58 de 1976). Durante 30 años, la Ley 58 definía a la persona que poseía el grado de consejería en rehabilitación como:

El profesional que, con conocimientos adecuados de la conducta y el desarrollo humano y de las instituciones sociales, utiliza los principios y técnicas de consejería en rehabilitación para proveerles a las personas incapacitadas servicios compatibles a sus necesidades de rehabilitación. (p. 1) 
La Ley 58, desde su inicio, presentó una cláusula de transición para que los profesionales que prestaban servicios de consejería a personas con necesidades especiales en el Programa de Rehabilitación Vocacional y que poseían un diploma profesional o habían iniciado estudios hacia el grado de maestría en consejería en rehabilitación pudieran completarlo en un periodo que no excediera los cuatro años a partir de la aprobación de la ley. Esto facilitó que no se interrumpieran servicios a personas con necesidades especiales y que los profesionales completaran los requisitos exigidos por ley. Con ello, se reconoce la profesión no solo en la academia sino en la práctica profesional.

\section{Creación de la primera Asociación de Consejeros en Rehabilitación}

Otro sector que contribuyó al desarrollo de la profesión, tanto en Estados Unidos como Puerto Rico, fueron las organizaciones profesionales. Previo a la creación de la primera organización de profesionales de la consejería en rehabilitación de Puerto Rico, en 1925 en los EUA, surge la National Rehabilitation Association (NRA) como consecuencia de la Ley Federal Smith- Fess Act de 1920 (Public Law No. 66-236, 1920). Esta organización agrupa a profesionales del campo de la rehabilitación, incluyendo a personal consejero en rehabilitación, de todos los Estados, Puerto Rico y demás territorios. Como organización, su fin es abogar y defender los derechos de las personas con necesidades especiales en el foro legislativo y comunitario, promover el desarrollo profesional de sus miembros a través de actividades de educación continua y comunitaria. También su misión es promover la ética y dar a conocer los últimos cambios en el campo de la rehabilitación.

En 1958 se crea la Asociación Nacional de Consejería en Rehabilitación (National Rehabilitation Counseling Association) en EUA y se une a la NRA como una División. Para el año 1959, un grupo de profesionales del campo de la rehabilitación se organiza en Puerto Rico y crea la Asociación Puertorriqueña de Rehabilitación (APR). Siguiendo las normas y procedimientos establecidos en el país, fue incorporada el 25 de junio de 1973 en el Departamento de Estado de Puerto Rico, como una organización sin fines de lucro por su primer presidente, el señor José Martínez Pacheco (Departamento de Estado, 1973). Se adscribió como un capítulo de la NRA. Un gran número de sus miembros en Puerto Rico eran consejeros en rehabilitación. La organización es disuelta el 3 de noviembre de 2011 debido a que la cantidad de miembros se redujo ya que las personas consejeras en rehabilitación se estaban agrupando en el Colegio de Profesionales de la Consejería en Rehabilitación (Departamento de Estado, 2011).

De forma concomitante, otro grupo surgía el 22 de marzo de 1969. Los profesionales de la consejería en rehabilitación de Puerto Rico también crearon su asociación, conocida como la Asociación de Consejeros en Rehabilitación de Puerto Rico (ACER) (Reglamento-Constitución, 2005). Esta es incorporada en el Departamento de Estado de Puerto Rico, como una organización sin fines de lucro, el 28 de marzo de ese mismo año, por su primer presidente, el señor Rubén Elí Matos (Departamento de Estado, 1969). Sus objetivos principales eran: 
URL: http://www.una.ac.cr/educare

CORREO: educare@una.cr

Establecer la colaboración y comunicación entre los consejeros en rehabilitación, las agencias públicas o entidades privadas ...; Enaltecer la profesión ...; Identificar, estudiar y analizar situaciones o asuntos que confrontan los consejeros en rehabilitación...; Estimular el más alto crecimiento profesional ... ; Realzar la imagen y desempeño profesional ... . ; Servir de instrumento representativo ante los organismos oficiales y de la comunidad y fomentar la participación activa de cada uno de sus miembros en actividades educativas y culturales.... Además promover la ética profesional, entre otros. (pp. 2-3)

Esta se mantuvo activa en el cumplimiento de sus propósitos y objetivos y en el desarrollo de actividades profesionales de mejoramiento y excelencia hasta que fue creado el Colegio de Profesionales de la Consejería en Rehabilitación de Puerto Rico. Entre sus gestas se destacan aspectos relacionados con condiciones laborales, mejoramiento de sueldos y actividades dirigidas a la unificación de la clase profesional. Es importante señalar que, desde la década de los ochenta (80), esta organización y sus miembros, reunidos en asamblea ordinaria y extraordinaria, aprobaron tres (3) mandatos que cambiarían el curso de la profesión. Estos incluían un plan para comenzar los trabajos hacia la colegiación de la profesión, con el objetivo de prestar servicios más eficientes, eficaces y de calidad que redundaran en beneficios y oportunidades de superación para las personas con necesidades especiales en Puerto Rico (Acta ACER, 1988). Partiendo de estos mandatos, se estableció el Comité Ad-hoc de Colegiación presidido por el doctor Ángel A. Villafañe Santiago, el cual más tarde se convirtió en el Comité de Asuntos Legislativos.

Este Comité, en conjunto con todos los integrantes de la Junta de Profesionales de la Consejería en Rehabilitación y otros colegas colaboradores, cabildearon en la Legislatura de Puerto Rico para radicar el Proyecto del Senado 2653 (2004) a los fines de crear la ley para comenzar los trabajos del Colegio de los Profesionales de la Consejería en Rehabilitación (CPCR). Este fue aprobado en el Senado de Puerto Rico pero al llegar a la Cámara de Puerto Rico, no pudo ser visto en las comisiones ya que era el último día de sesión debido a que se entraba a la veda del periodo eleccionario para elegir al nuevo gobierno que llevaría los destinos de Puerto Rico por los próximos cuatro años.

No fue sino hasta dos años más tarde, en el 2006, cuando se pudo retomar nuevamente el proyecto y se convirtió en el Proyecto de la Cámara 1518 (2006). En la exposición de motivos se amplió su justificación legal tomando en consideración las exigencias de la Ley Pública 101-336 de 1990, conocida como "American with Disabilities Act" (ADA); la Ley Pública 93-112 de 1973, según enmendada, conocida como "Ley de Rehabilitación"; la Ley Núm. 97 de 6 de junio de 2000, Ley de Rehabilitación Vocacional de Puerto Rico, según enmendada; la Ley Pública 101-476 del 30 de Octubre de 1990, según enmendada, conocida como "Individuals with Disabilities Educational Act"; la Ley Núm. 51 de 7 de junio de 1996, conocida como "Ley de Servicios Educativos Integrales para Personas con Necesidades Especiales de Puerto Rico"; la Ley Pública 105-220 de 7 de agosto 1998, conocida como "Workforce Investment Act" (WIA); la Ley Pública 100-690 de 18 de 
noviembre de 1988, conocida como "Drug-Free Workplace Act"; la Ley Pública 105-394 de 13 de noviembre de 1998, conocida como "Assistive Technology Act (AT-ACT) de 1998", Sección [215]; la "Hearing Aid Compatibility Act de 16 de agosto de 1988", y la "Television Decoder Circuitry Act de 15 de octubre de 1990" y otras leyes relacionadas con la protección de los derechos civiles, con la igualdad de oportunidades y de acceso a servicios y calidad de vida para las personas con necesidades especiales. Con este marco legal se consideró de interés público la creación, por Ley, del "Colegio de los Profesionales de la Consejería en Rehabilitación de Puerto Rico", y el establecimiento del requisito de colegiación obligatoria.

El cabildeo fue extenso, pero produjo su aprobación al convertirse en la Ley 160 del 16 de agosto de 2006. Esta disponía una consulta a los "Profesionales de la Consejería en Rehabilitación de Puerto Rico" para determinar la constitución del "Colegio de Profesionales de la Consejería en Rehabilitación de Puerto Rico", y establecer el requisito de colegiación obligatoria; especificar sus propósitos y facultades; determinar su reglamentación; y fijar sanciones por el ejercicio de la Consejería en Rehabilitación en contravención de esta Ley.

En septiembre de 2006, la Comisión, creada en virtud de la Ley 160 de 16 de agosto (2006), compuesta por veinticuatro (24) miembros (todos profesionales de consejería en rehabilitación), realizó su plan de trabajo que incluía la visita a todo Puerto Rico para orientar sobre el futuro colegio, al personal de consejería en rehabilitación, enviar las boletas de votación y un boletín informativo que contenía los artículos de mayor relevancia. El propósito fue concienciar a este personal sobre la "Consulta de Colegiación".

Para 2 de junio de 2007, se llevó a cabo la primera convocatoria a los fines de constituir el Colegio de los Profesionales de la Consejería en Rehabilitación, en la Universidad Interamericana de Puerto Rico, Recinto (Dependencia) de Cupey. La asistencia no cumplió con el quórum requerido debido a que la Ley 160 de 16 de agosto (2006) estipulaba lo siguiente:

Se requerirá la participación del cincuenta (50) por ciento de los Consejeros en Rehabilitación licenciados y certificados como activos por la Junta. Así también, para la aprobación de la colegiación obligatoria se requerirá el voto afirmativo del cincuenta por ciento de los participantes en la votación que se realizara a tal fin. (p. 2)

Al no cumplir con este requisito hubo que llevar a cabo una segunda convocatoria en la Universidad de Puerto Rico, Recinto de Río Piedras, para el 25 de agosto de 2007, para los mismos propósitos. En esta ocasión se sobrepasó el quórum establecido por la Ley, que establecía:

En el caso de que para la primera consulta no se logre el porcentaje de participación requerido y una mayoría se haya expresado a favor de la colegiación, la Comisión de Consulta escogerá una nueva fecha para comenzar una segunda consulta con iguales propósitos y cuya participación mínima requerida será el cuarenta (40\%) por ciento de 
URL: http://www.una.ac.cr/educare

CORREO: educare@una.cr

los consejeros en rehabilitación licenciados y certificados como activos por la Junta. Así también, para la aprobación de la colegiación obligatoria en una segunda consulta, se requerirá el voto afirmativo de cincuenta (50) por ciento de los participantes en la votación. De no lograrse nuevamente el por ciento de participación requerido en esta Ley, se entenderá que los consejeros en rehabilitación rechazan la colegiación compulsoria y la Comisión de Consulta no tendrá facultad para realizar una nueva consulta para los fines autorizados en esta Ley. (p. 2)

El hecho que se lograra el quórum en la segunda convocatoria dio paso a la primera reunión de la Asamblea Constituyente del Colegio de los Profesionales de la Consejería en Rehabilitación. En esta reunión se aprueba la cuota a cobrar a los colegiados y se designó un comité para preparar el reglamento de la organización, el cual sería sometido a la consideración de la matrícula, para su aprobación, en la segunda reunión de la Asamblea Constituyente. Aprobado el Reglamento, se constituye legalmente el Colegio de Profesionales de la Consejería en Rehabilitación el 28 de marzo de 2008 y comienza el proceso de transición para la disolución de la ACER de Puerto Rico, proceso que se completó el 30 de junio de 2008 (Departamento de Estado 2008, agosto 6). Con este esfuerzo se le dio continuidad a ese grupo de hombres y mujeres que hicieron de la ACER de Puerto Rico el organismo de vanguardia que representaba dignamente a la profesión en nuestra isla y que sirvió de puente para una nueva etapa en la profesión. En esta nueva gesta el grupo de profesionales fue guiado por la sabia dirección de su primer presidente, el Lcdo. José Lionel Velázquez Altagracia.

\section{Esfuerzos legislativos posteriores: Respondiendo a la época y a nuevos retos}

Mientras se luchaba por la aprobación del Colegio de Profesionales de la Consejería en Rehabilitación, los miembros del "Comité de Asuntos Legislativos" de la ACER de Puerto Rico habían estado analizando la Ley 147 del 9 de agosto de 2002, Ley para Reglamentar la Consejería Profesional en Puerto Rico y el impacto que tendría para la clase profesional debido a que la Ley 58 de 1976, según enmendada, enmarcaba al consejero en rehabilitación como proveedor de servicios a personas con necesidades especiales exclusivamente; aun cuando se encontraba trabajando en otros escenarios laborales. Por tanto, se presenta ante la Legislatura de Puerto Rico otro proyecto de ley (Proyecto de la Cámara 1517) que más tarde se convirtió en la "Ley 198 del 11 de septiembre de 2006".

El propósito fue enmendar los Artículos 2, 3, 4 y 8; y añadir un nuevo Artículo 7-A a la Ley Núm. 58 de 27 de mayo de 1976, según enmendada, conocida como“Ley para Reglamentar la Profesión de Consejería en Rehabilitación en Puerto Rico", a los fines de redefinir el concepto de "consejería en rehabilitación"; para añadir la definición del concepto de "vida independiente"; para separar a la Junta creada en esta Ley del Departamento de Estado y adscribirla al Departamento de Salud; establecer la obligatoriedad de los profesionales de 
la consejería en rehabilitación de cursar estudios continuos; establecer un Código de Ética de los Profesionales de la Consejería en Rehabilitación; y para otros fines relacionados. (p. 1)

Entre estos se encuentran la inclusión de las diez áreas de conocimiento o competencias en donde el profesional de la consejería en rehabilitación se desarrollaba académicamente y laboralmente:

(1) Identidad Profesional; 2) Diversidad Social y Cultural; 3) Desarrollo y Crecimiento Humano; 4) Desarrollo de Carreras y Empleo; 5) Consejería y Consultoría; 6) Trabajo de Grupos; 7) Avalúo; 8) Investigación y Evaluación de Programas; 9) Aspectos Médicos Funcionales y Ambientales de la Incapacidad; y 10) Recursos y Servicios de Rehabilitación. (p. 2)

Con este aporte se reconoce que el personal de consejería en rehabilitación tiene la pericia y los conocimientos necesarios para trabajar con personas con necesidades especiales y sin estas, tanto en consejería individual como grupal. Por otro lado, en la redefinición de la consejería en rehabilitación se amplía el concepto a tono con las nuevas tendencias del Siglo $\mathrm{XXI}$ y en concordancia con los nuevos mandatos legislativos que señalan:

Significa un proceso abarcador e individualizado o grupal de naturaleza estructurada y facilitadora que establece una relación interaccionar entre el consejero en rehabilitación y la persona con o sin limitaciones funcionales para el desarrollo integral de sus habilidades y destrezas orientado hacia todos los aspectos de su vida incluyendo sus metas de empleo o de una vida independiente para alcanzar su óptima calidad de vida. Este proceso está dirigido hacia el desarrollo o la restauración de la independencia funcional y la calidad de vida del ser humano. La independencia funcional que se persigue mediante el proceso de consejería en rehabilitación involucra varias metas que conllevan inclusión, autosuficiencia, integración y vida autónoma. Incluye: altos índices de calidad de vida que sean el resultado que se alcance como parte de la rehabilitación integral de este ser humano. Esto constituye la oportunidad de incluir unas dimensiones significativas y consideraciones particulares en la vida del ser humano tales como: la médica, la psicológica, la social personal, cultural, educativa, vocacional y la espiritual. (p. 3)

De esta forma se convirtió en la enmienda más importante realizada a la Ley 58 , supra en treinta (30) años. 
URL: http://www.una.ac.cr/educare

CORREO: educare@una.cr

Es importante señalar que, durante el proceso de operacionalizar la Ley 160 del 16 de agosto (2006), el Comité de Asuntos Legislativos se percata de que en esta Ley había unos aspectos que requerían enmendarse y redactó un documento que luego se convirtió en el Proyecto de la Cámara 3078 y rápidamente en la Ley 214 del 2007. Su propósito fue:

Enmendar el inciso (b) y (c) del Artículo 2 y los Artículos 4 y 7 de la Ley Núm. 160 de 16 de agosto de 2006, conocida como "Ley del Colegio de los Profesionales de la Consejería en Rehabilitación de Puerto Rico", a los fines de atemperar los mismos a la legislación vigente. El artículo 2 define que la sede oficial del "Colegio de los Profesionales de la Consejería en Rehabilitación de Puerto Rico", estará ubicada donde los colegiados así lo decidan a través de su Junta de Directores. (p. 1)

También enmienda el Artículo 7 para establecer los requisitos para pertenecer al Colegio; indicando que:

Pertenecerán al Colegio toda persona que posea una licencia expedida por la Junta que le autorice a ejercer la profesión de Consejería en Rehabilitación en Puerto Rico, conforme a lo establecido en la Ley Núm. 58, supra. Todo profesional de la Consejería en Rehabilitación deberá tener vigente la licencia y haber cumplido con los requisitos de certificación, 0 de re-certificación que le sean aplicables. Pertenecerán al Colegio todo consejero en rehabilitación que trabaje o preste servicios sin paga en áreas relacionadas a la conducta humana y que dependiendo de la visión y misión de la agencia, organización o entidad tenga título como: Consejeros en Rehabilitación, Manejador de Casos, Orientadores, Consejeros, Consejero Vocacional, Consejero Evaluador, Especialista en Rehabilitación, Asesores en organizaciones públicas, privadas o en organizaciones no gubernamentales de base comunitaria, sin que ello se entienda como una limitación. (p. 4)

Otro esfuerzo legislativo fue el Proyecto de la Cámara 2719 (2006), el cual buscaba que la Junta de Consejeros Profesionales de Puerto Rico reconociera las áreas de conocimiento esbozadas en la Ley 198 del 11 de septiembre (2006) donde las primeras ocho (8) eran similares a las de consejería profesional y la pericia del consejero en rehabilitación, con lo que otorgaba la Licencia de Consejero Profesional. En el proceso de análisis del proyecto legislativo, la Comisión del Trabajo y Relaciones Laborales de la Cámara de Representantes de Puerto Rico cita a una "Conferencia Legislativa" al presidente de la Junta Examinadora de Profesionales de la Consejería en Rehabilitación y al presidente de la Junta Examinadora de Consejeros Profesionales para discutir aspectos relacionados al proyecto. En medio de esta dinámica legislativa, la Cámara de Representantes entendió meritorio insertar la discusión de los propósitos de la presente medida discutiéndolos e incorporando los mismos en la evaluación de los Proyectos del Senado 837, 1499 y 1955, según atendidos por la Comisión de 
Gobierno y la comisión informante. El 24 de noviembre de 2008, la Cámara de Representantes no recomienda la medida. Es importante aclarar que el Proyecto del Senado 1499 pretendía:

Enmendar el inciso f) del Artículo 8, añadir un nuevo inciso (C) al Artículo 15 y enmendar el Artículo 16 de la Ley 147 de 9 de agosto de 2002, según enmendada, conocida como "Ley para Reglamentar la Práctica de la Consejería Profesional en Puerto Rico", a los fines de incluir el grado de maestría en consejería en rehabilitación como requisito para obtener la licencia en consejería profesional; fijar sanciones administrativas y penales a las Instituciones, Agencias o Instrumentalidades Gubernamentales y Privadas que permitan el ejercicio de la profesión de consejería sin licencia según requerida por esta Ley, y para otros fines. (p. 1)

Este esfuerzo legislativo requirió de múltiples reuniones con los profesionales de la consejería en rehabilitación, dada a la situación de que había compañeros que, por muchos años, ya tenían permanencia en los distintos sistemas educativos del país (secundario y postsecundario) y estaban operando sin licencia con el título de "orientadores, consejeros o manejadores de casos, entre otros", debido a que la profesión de orientación y consejería no estaba reglamentada hasta que se aprueba la Ley 147 el 9 de agosto de 2002 y su nombre cambia a consejería profesional. En su Artículo 16 (a) indica:

Ninguna cláusula de esta Ley se aplicará a las actividades y a los servicios prestados por personas cualificadas para otras profesiones, como por ejemplo médicos, trabajadores sociales, consejeros en rehabilitación o sicólogos que practican la consejería de acuerdo con las leyes de Puerto Rico. (p. 12)

Por lo tanto, dentro de su cláusula de transición no se contempló a los profesionales de la consejería en rehabilitación, por lo que quienes no tenían licencia estaban fuera de ambas leyes.

A esos efectos, el Comité de Asuntos Legislativos (2009), en la primera asamblea del Colegio de los Profesionales de la Consejería en Rehabilitación, somete a consideración un ante proyecto de ley cuyo propósito fue que se le permitiera a aquellos profesionales de la consejería en rehabilitación, con permanencias en sus trabajos y más de diez años de experiencia, que no aprobaran la reválida con 67 puntos (el mínimo establecido por la Junta Examinadora de Consejeros/as en Rehabilitación era 70) se les permitiera tomar unas educaciones continuas en las áreas no aprobadas. Este anteproyecto permitía un proceso de transición al cambio de la ACER al CPCR, al permitir licenciar a estos profesionales con vasta experiencia dentro de las diversas áreas de la consejería y tendría un efecto directo en aumentar el número de colegiados al Colegio de los Profesionales de la Consejería en Rehabilitación. El mismo fue derrotado por lo que no pasó al trámite legislativo de Puerto Rico (CPCR, 2010). 
URL: http://www.una.ac.cr/educare

CORREO: educare@una.cr

Para este ante proyecto de ley se habían analizado los diferentes escenarios laborales donde por más de treinta años los profesionales de la consejería en rehabilitación en Puerto Rico se mantenían prestando servicios. Algunos de estos fueron: Departamento de Educación, la Corporación del Fondo del Seguro del Estado, organizaciones de base comunitaria, Administración de Servicios de Salud Mental y Contra la Adicción, consultoría, oficinas de clínica privada, Oficina del Seguro Social por Incapacidad, Sistema de Tribunales, Oficina de Asuntos para la Vejez, Administración de Rehabilitación Vocacional y servicios de peritos en el sistema judicial, mejor conocido como consejería forense, centros de rehabilitación, centros de salud mental público y privados y hospitales psiquiátricos, entre otros. Sin embargo, es a través de la redefinición de la consejería en rehabilitación en la Ley 198 del 11 de septiembre (2006) y la constitución del Colegio de los Profesionales de la Consejería en Rehabilitación que se reconoce, desde el punto de vista legal, el alcance de la práctica profesional en múltiples ámbitos; no así para el campo de la salud mental en Puerto Rico. Este dato histórico es de mucha relevancia, porque aunque parece contradictorio, la realidad histórica es que los Consejeros en Rehabilitación no estábamos en la Ley de Salud Mental de Puerto Rico, conocida como Ley 408 de 2000.

Por esto, para el año 2009, en la Escuela Graduada de Consejería en Rehabilitación de la Universidad Central de Bayamón, se inicia el proceso de enmendar la Ley 408 de 2000 mejor conocida como Ley de Salud Mental de Puerto Rico, a través del primer grupo de estudiantes comandado por el doctor Ángel A. Villafañe Santiago. Este esfuerzo es adoptado por el Comité de Asuntos Legislativos del Colegio de los Profesionales de la Consejería en Rehabilitación y, posteriormente, por la Junta Examinadora de Consejeros en Rehabilitación, la Escuela Graduada de Consejería en Rehabilitación de la Universidad de Puerto Rico y la Escuela Graduada de la Pontificia Universidad Católica de Puerto Rico. Finalmente, el anteproyecto de ley fue adoptado por el Senado de Puerto Rico y se convirtió en el Proyecto del Senado 1972 por petición, a los fines de enmendar los siguientes artículos e incisos: añadir un nuevo inciso (o) al Artículo 1.06 y enmendar los Artículos 2.18, 2.19, 4.03, 6.01, 8.06, 8.11, 10.01 y 10.2 de la Ley 408 de 2000, según enmendada, conocida como "Ley de Salud Mental de Puerto Rico".

El propósito principal fue incluir al personal de consejería en rehabilitación como parte del grupo de profesionales que prestan servicios de salud mental en Puerto Rico. Este proyecto fue aprobado en el Senado de Puerto Rico y continuó el trámite legislativo para la Cámara de Representantes de Puerto Rico, pero en este cuerpo no fue aprobado. Sin embargo, los esfuerzos del Colegio de los Profesionales de la Consejería en Rehabilitación y de la Junta Examinadora, conjuntamente con las aportaciones del doctor Ángel Villafañe Santiago, el doctor Ernesto Pérez Cartagena y otras personas colegas de la profesión lograron traer nuevamente el proyecto a votación a través del Proyecto del Senado 735, el cual fue finalmente aprobado convirtiéndose en la Ley 170 del 16 de agosto de 2012. Con este esfuerzo legislativo el profesional de la consejería en rehabilitación queda reconocido y autorizado desde el punto de vista legal para continuar la prestación de servicios a aquellas personas con condiciones mentales en centros de rehabilitación y hospitalización psiquiátrica, práctica pública y privada y en otros escenarios donde los servicios de este profesional sean necesarios. 
Para concluir, podemos señalar que el 13 de marzo de 2013, el Congreso de los EUA emitió la Resolución del Senado 106 del Senado, para designar el 22 de marzo como el Día de Apreciación Nacional de los Consejeros en Rehabilitación (National Rehabilitation Counselors Appreciation Day). Este reconocimiento, extensivo al personal de consejería en rehabilitación de Puerto Rico, fue gestionado por la National Rehabilitation Association (NRA), lo que constituye otro aporte al reconocimiento de la profesión de la consejería en rehabilitación en nuestra sociedad y resalta la importancia de tener este profesional en los equipos de trabajos en los distintos escenarios que prestan servicios a las personas con necesidades especiales o sin ellas, en Puerto Rico.

\section{Conclusión}

La consejería en rehabilitación es una profesión que emergió de la necesidad de atender a los soldados que venían con diferentes necesidades especiales de la Primera Guerra Mundial y que luego, gracias a proyectos legislativos federales y estatales, fueron extendidos a una creciente población de civiles. Esto requirió de la preparación de profesionales especializados con un conocimiento diferente para entender las limitaciones funcionales y las diferentes manifestaciones de estas. Además, el estudio y tratamiento del impacto psicosocial de las personas con necesidades especiales, el ajuste a la condición, evaluación y desarrollo del potencial residual, consejería personal y de carreras, la eliminación de barreras actitudinales y arquitectónicas. Estas son solo algunas de las funciones y servicios que el personal de consejería en rehabilitación históricamente ha ofrecido a las personas con necesidades especiales en EUA y Puerto Rico desde principios del Siglo XX.

Es una profesión de vanguardia, única en su clase, que ha evolucionado con el tiempo y ante la diversidad de las condiciones con las que trabaja. El desarrollo tecnológico ha ampliado sus áreas de competencia, integrando la asistencia tecnológica para mantener, mejorar o retener funciones que ayudarán, a las personas con necesidades especiales, a ser más independientes e incorporarlas al mundo del trabajo, conjuntamente con los acomodos razonables como recurso en el escenario laboral, para que la persona pueda cumplir con las tareas esenciales de los puestos de trabajo, entre otros.

Como se ha ilustrado en este recuento histórico/teórico, la profesión de consejería en rehabilitación, además de los cambios en las regulaciones federales y estatales, desde su inicio también ha estado en continúa revisión de su currículo y las exigencias académicas hasta lograr el grado de maestría en consejería en rehabilitación en Puerto Rico. Estos esfuerzos legislativos, colaborativos y de gran envergadura para la clase profesional han respondido a las necesidades de las personas con necesidades especiales, a los reclamos y necesidades de los colegiados, otorgándole un sitial de reconocimiento a la profesión y al trabajo encomiable realizado en todo Puerto Rico por excelentes profesionales del campo. 
URL: http://www.una.ac.cr/educare

CORREO: educare@una.cr

Estos seres humanos, hombres y mujeres, comprometidos con el proceso de rehabilitación, han fiscalizado y promovido piezas legislativas, desarrollo económico y movimientos históricos que han permitido una prestación de servicios a las personas con necesidades especiales (Romañach y Lobato, 2005) que redunda en una mejor calidad de vida para estas, servicios educativos, vocacionales y de empleo alineados con su capacidad residual e independencia económica del Estado, entre otros. De esta manera, Puerto Rico se coloca a la vanguardia, al tener profesionales de la consejería en rehabilitación ofreciendo servicios cobijados por las leyes que le dan protección a la persona con necesidades especiales. Al mismo tiempo, incorpora, desde el punto de vista legal, a este profesional de la salud que es tan versátil y que puede trabajar tanto en el sector público como privado desde enfoques inter, multi y transdisciplinario con personas con necesidades especiales físicas, mentales, sensoriales y sociales.

Sin embargo, el trabajo no termina aquí; esta revisión permite reevaluar hacía dónde se va. El camino es claro, hay otros retos que aún hay que enfrentar; el mercadeo de la profesión entre los paraprofesionales todavía está en etapas iniciales, previamente identificadas por Marín, Ríos y Santiago (1995); se debe continuar educando a los prospectos patronos y empresarias sobre la potencia de las personas con necesidades especiales y ampliar el radio de acción a otros sistemas de prestación de servicios a nivel privado. La población de personas con necesidades especiales está creciendo a nivel mundial; lo que implica que podría convertirse en uno de los grupos minoritarios más grande a nivel mundial que necesitará la ayuda de más profesionales de la consejería en rehabilitación en Puerto Rico y EUA. Es menester continuar la educación social, el desarrollo de capital humano y el desarrollo de centros de servicios para atender esa demanda creciente de servicios.

\section{Referencias}

American Medical Association (2008). Health Care Career. Recuperado de http://www.ama-assn. org/ama1/pub/upload/mm/40/rehabcoun0809.pdf

Americans With Disabilities Act, as amended [Acta de los americanos con impedimentos, según enmienda], Pub. L. No. 101-336, 104 Stat. 327 (1990).

Asociación de Consejeros en Rehabilitación de Puerto Rico Inc. (1988). Acta. San Juan Puerto Rico.

Asociación de Consejeros en Rehabilitación de Puerto Rico Inc. (2005). Reglamento-Constitución, Artículo Il-Objetivos Generales, San Juan, Puerto Rico.

Assistive Technology Act [Acta de Asistencia Tecnológica], Pub. L. No. 105-394, 112 Stat. 3656, 29 USC 3035, Section 215 (1998).

Colegio de los Profesionales de la Consejería en Rehabilitación (2009). Informe del Comité de Asuntos Legislativo, Ponce, Puerto Rico. 
Colegio de los Profesionales de la Consejería en Rehabilitación (2010). Acta Primera Asamblea, Mayagüez: Puerto Rico.

Departamento de Estado (1969). Registro de Corporaciones: Asociación de Consejeros en Rehabilitación, incorporada, registro 5127. Estado Libre Asociado de Puerto Rico. Recuperado de www.estado.gobierno.pr

Departamento de Estado (1973). Registro de corporaciones: Asociación Puertorriqueña de Rehabilitación, incorporada, registro 6869. Estado Libre Asociado de Puerto Rico. Recuperado de www.estado.gobierno.pr

Departamento de Estado (2008, agosto 6). Registro de Corporaciones: Certificación de Disolución de la Asociación de Consejeros en Rehabilitación incorporada, registro 5127. Estado Libre de Puerto Rico.

Departamento de Estado (2011, noviembre 3). Registro de Corporaciones: Certificación de Disolución de la Asociación Puertorriqueña de Rehabilitación Incorporada, registro 6889. Estado Libre de Puerto Rico.

Drug Free Workplace Act [Acta de lugares de trabajo libres de drogas], Pub. L. No. 100-690, 102 Stat. 4181 (1988).

Hearing Aid Compatibility Act [Acta de compatibilidad de ayuda auditiva], Pub. L. No. 100-394 102 Stat. 976 [47 U.S.C. 610 (b)] (1988).

History y Regulations Legislative History of the American State-Federal Vocational Rehabilitation (VR) Program (1917). Smith-Hughes Act. [Acta de Smith-Hughes]. Recuperado de www. in.gov/fssa/files/History and Regulations.pdf

Individuals With Disabilities Education Act, as amended [Acta de Educación de Individuos con Impedimentos, según enmienda], Pub. L. No. 101-476, 104 Stat. 1142 (1990).

Individuals with Disabilities Education Act (IDEA ) ... An overview of the Individuals with Disabilities Education Act (IDEA). [Una mirada del Acta de Educación de Personas con Impedimentos] Recuperado http://www.education.com/reference/article/individualsdisabilities-education-act/

Ley 28 de 1931. Ley de Enseñanza Vocacional. (1931).

Ley Núm. 414 de 1947. Ley de Rehabilitación Vocacional de Puerto Rico. (1947).

Ley Núm. 58 de 1976. Para reglamentar la práctica de la consejería en rehabilitación en Puerto Rico (1976).

Ley Núm. 51 de 1996. Ley de Servicios Educativos Integrados para Personas con necesidades especiales en Puerto Rico (1976).

Ley Núm. 97 de 2000. Ley de Rehabilitación Vocacional de Puerto Rico (2000). 
URL: http://www.una.ac.cr/educare

CORREO: educare@una.cr

Ley Núm. 408 de 2000. Ley de Salud Mental de Puerto Rico (2000).

Ley Núm. 147 de 2002. Para reglamentar la práctica de la Consejería Profesional en Puerto Rico (2002).

Ley Núm. 160 de 2006. Para disponer respecto a la constitución del "Colegio de Profesionales de la Consejería en Rehabilitación de Puerto Rico", y establecer el requisito de colegiación obligatoria; especificar sus propósitos y facultades; determinar su reglamentación; y fijar sanciones por el ejercicio de la Consejería en Rehabilitación en contravención de esta Ley (2006).

Ley Núm. 198 de 2006. Para enmendar los arts. 2, 3, 4, 8 y añadirArt. 7-A a la Ley Núm. 58 de 1976: Ley para Reglamentar la Profesión de Consejería en Rehabilitación en Puerto Rico. (2006).

Ley Núm. 214 de 2007. Para enmendar los arts. 2, 4 y 7 de la Ley Núm. 160 de 2006: Ley del Colegio de los Profesionales de la Consejería en Rehabilitación (2007).

Ley Núm. 170 de 2012. Para añadir un nuevo inciso (o) y reenumerar los incisos (o) a (www) como (p) a (xxx) al Artículo 1.06 y enmendar los Artículos 2.18, 2.19, 4.03, 6.01, 8.06, 8.11, 10.01 y 10.2 de la Ley 408-2000, según enmendada, conocida como "Ley de Salud Mental de Puerto Rico", a los fines de incluir a los consejeros en rehabilitación como parte del grupo de profesionales que prestan servicios de salud mental en Puerto Rico (2012).

Marín, A., Ríos, E. y Santiago, B. (1995). La profesión de consejería en rehabilitación y su trayectoria en Puerto Rico. Ponencia presentada en la Vigesimosexta Convención Anual de la Asociación de Consejeros en Rehabilitación de Puerto Rico. Caguas, Puerto Rico.

Parker, R. y Szymanski, E. (1998). Philosophical, Historical and Legislative Aspects of the Rehabilitation Counseling Profession. Rehabilitation Counseling: Basics and Beyond [Consejería en rehabilitación: origen y más allá]. ( $3^{\mathrm{a}}$ ed., pp. 1-3). Austin, Texas: Pro-Ed.

Parker, R., Szymanski, E. y Patterson, J. (2005). Rehabilitation Counseling: Basics and Beyond [Consejería en rehabilitación: Origen y más allá]. (4ª ed.). Austin, Texas: Pro-Ed.

Proyecto de la Cámara 1517. Para enmendar los Artículos 2, 3 y 8 de la Ley Núm. 58 de 27 del mayo de 1976; actualizando la definición de los conceptos de rehabilitación y vida independiente para que sean cónsonos con la óptima calidad de vida, añadir la educación continuada para que los consejeros en rehabilitación se mantengan actualizados en sus conocimientos relacionados a la profesión de la consejería en rehabilitación para contribuir al pleno bienestar y óptimo desarrollo de las personas con necesidades especiales de Puerto Rico y transferir la Junta Examinadora de Consejeros en Rehabilitación del Departamento de Estado al Departamento de Salud del Estado Libre Asociado de Puerto Rico (2005). 
Proyecto de la Cámara 1518. Para disponer respecto a la constitución del Colegio de Profesionales de la Consejería en Rehabilitación, con el requisito decolegiación obligatoria; especificar sus propósitos y facultades; determinar su reglamentación; y fijar sanciones por el ejercicio de la Consejería en Rehabilitación en contravención de esta ley (2006).

Proyecto de la Cámara 2719. Para enmendar los Artículos 8 y 16 de la Ley Núm.147 de 9 de agosto de 2002, conocida como la "Ley que Reglamenta la Práctica de la Consejería Profesional en Puerto Rico. Oficina de Asuntos Legislativos, trámite legislativo (2006). Recuperado de http://www.oslpr.org/buscar/

Proyecto de la Cámara 3078. Para enmendar el inciso (c) del Artículo 2 y los Artículos 4, 7 y 14 incisos (f) y (h) de la Ley Núm. 160 de 16 de agosto de 2006, conocida como "Ley del Colegio de los Profesionales de la Consejería en Rehabilitación de Puerto Rico", a los fines de atemperar los mismos a la legislación vigente (2006).

Proyecto de la Cámara 3078. Para enmendar los arts. 2, 4 y 7 de la Ley Núm. 160 de 2006: Ley del Colegio de los Profesionales de la Consejería en Rehabilitación (2007).

Proyecto del Senado 1499. Para enmendar el inciso f) del Artículo 8, añadir un nuevo inciso (C) al Artículo 15 y enmendar el Artículo 16 de la Ley 147 de 9 de agosto de 2002, según enmienda, conocida como "Ley para Reglamentar la Práctica de la Consejería Profesional en Puerto Rico", a los fines de incluir el grado de maestría en consejería en rehabilitación como requisito para obtener la licencia en consejería profesional fijar sanciones administrativas y penales a las instituciones, agencias o instrumentalidades gubernamentales y privadas que permitan el ejercicio de la profesión de consejería sin licencia según requerida por esta ley, y para otros fines. El Capitolio, San Juan, Puerto Rico (2006).

Proyecto del Senado 2653. Para disponer respecto a la constitución del Colegio de Profesionales de la Consejería en Rehabilitación de Puerto Rico, con el requisito de colegiación obligatoria; especificar sus propósitos y facultades; determinar su reglamentación; y fijar sanciones por el ejercicio de la Consejería en Rehabilitación en contravención de esta ley. (2004)

Rehabilitation Act of 1973 [Acta de Rehabilitación de 1973], Pub. L. No. 93-11287, Stat. 355, 29 U.S.C. 701 (1973).

Riggar, T. F. y Maki, D. R. (Eds.) (2004). Handbook of Rehabilitation Counseling [Manual de consejería en rehabilitación]. New York: Springer Publishing Co.

Romañach, J. y Lobato, M. (mayo, 2005). Diversidad funcional, nuevo término para la lucha por la dignidad en la diversidad del ser humano. (Foro de Vida Independiente). Recuperado de http://www.imagina.org/archivos/archivos vi/Diversidad\%20funcional vf.pdf 
URL: http://www.una.ac.cr/educare

CORREO: educare@una.cr

Rubin, S. y Roessler, R. (2008). Foundations of the Vocational Rehabilitation Process [Principios de los procesos de rehabilitación vocacional] ( $6^{\mathrm{a}}$ ed.). Austin, Texas: Pro-Ed.

Senate Resolution 106-Commending Rehabilitation Counselors and Supporting the Goals and Ideals National Rehabilitation Counselors Appreciation Day Resolución del Senado 106Elogiando y apoyando las metas e ideales de la Asociación Nacional de Rehabilitación y Día del Consejero en Rehabilitación], 113th Congressional Record-Senate, S2902 (2013).

Smith-Fess Act [Acta Smith Fees], Pub. L. No. 66-236, 41 Stat. 735 (1920).

Soldier's Rehabilitation Act (Smith-Sears Act) [Acta de Rehabilitación de los Soldados (Acta Smith-Sears)], Pub. L. No. 66-11, Sess. 2, ch 107, 40 Stat. 617 (1918).

Television Decoder Circuitry Act [Acta de Decodificador de Circuitos de Televisión], Pub. L. No. 101-431, 104 Stat.960, [47 U.S.C. 303(u)] (1990).

Wagner-Payser Act [Acta Wagner-Payser], Pub. L. No. 73-3048 Stat. 113 (1933).Workforce Investment Act, as amended [Acta de Inversión a la Fuerza Trabajadora, según enmendada], Pub. L. No. 105-220, 112 Stat. 936 (1998).

Cómo citar este articulo en APA:

Villafañe, A. A. y Velázquez, J. L. y Báez, M. (2013). Evolución de la consejería en rehabilitación en Puerto Rico: Esfuerzos organizacionales y proyectos legislativos. Revista Electrónica Educare, 17(3), 117 136. Recuperado de http://www.revistas.una.ac.cr/index.php/EDUCARE/issue/current

Nota: Para citar este articulo en otros sistemas puede consultar el hipervinculo "Como citar el articulo" en la barra derecha de nuestro sitio web:

http:/www.revistas.una.ac.cr/index.php/EDUCARE/index 